Ambiente \& Água - An Interdisciplinary Journal of Applied Science
ISSN 1980-993X - doi:10.4136/1980-993X
www.ambi-agua.net
E-mail: ambi-agua@agro.unitau.br

\title{
Análise de tendência em séries históricas de vazão e precipitação: uso de teste estatístico não paramétrico
}

\author{
doi: 10.4136/ambi-agua.1427
}

Received: 16 Jun. 2014; Accepted: 07 Nov. 2014

\author{
Eduardo Morgan Uliana ${ }^{1 *}$; Demetrius David da Silva ${ }^{2}$; Estevão Morgan Uliana ${ }^{3}$; \\ Beatriz Silveira Rodrigues ${ }^{2}$; Lucas de Paula Corrêdo \\ ${ }^{1}$ Universidade Federal de Mato Grosso (UFMT), Sinop, MT, Brasil \\ ${ }^{2}$ Universidade Federal de Viçosa (UFV), Viçosa, MG, Brasil \\ ${ }^{3}$ Instituto Federal do Espírito Santo (IFES), Colatina, ES, Brasil \\ "Autor correspondente: e-mail: morganuliana@gmail.com, \\ demetrius@ufv.br, estevaomorgan@hotmail.com, \\ biasrodrigues23@hotmail.com, lucascorredo@gmail.com
}

\section{RESUMO}

A detecção de tendência em séries de vazão e precipitação apresenta expressiva importância para a gestão de recursos hídricos e ela pode ser feita por meio de testes estatísticos não paramétricos. O objetivo do trabalho foi avaliar a significância das tendências em série temporais de vazões e precipitações mensal e anual, por meio do teste de Pettitt. Foram utilizadas séries históricas de precipitações e vazões correspondentes ao período de 1939 a 2005, registradas nas estações 02041003 e 57450000, pertencentes à rede hidrometeorológica da Agência Nacional de Águas (ANA), localizadas no município de Alegre-ES. Foi possível identificar, com o teste de Pettitt, mudança de tendência na vazão média mensal do mês de outubro a partir do ano 1963. A vazão média deste mês aumentou $34,2 \%$ a partir do ano 1963. Já a série de precipitações apresentou tendência positiva nos meses de agosto e setembro a partir dos anos 1967 e 1964, respectivamente. O aumento da vazão média do mês de outubro pode ser consequência do aumento da precipitação nos meses de agosto e setembro, havendo a necessidade de estudos adicionais que levem em consideração a influência de fatores climáticos e antrópicos neste componente do ciclo hidrológico. Após a análise dos resultados obtidos concluiu-se que o teste de Pettit apresentou desempenho satisfatório para a detecção de tendências nas séries históricas de vazões e de precipitações.

Palavras-chave: chuva, estacionariedade, série temporal, teste de Pettitt, vazão média.

\section{Trend analysis of an historical series of flow and precipitation using a non-parametric statistical test}

\section{ABSTRACT}

Detecting trends in flow and precipitation series has great importance for the management of water resources and can be accomplished using statistic non-parametric tests. This work evaluated the significance of trends in flow and temporal series of precipitation as well as monthly and annual precipitation trends using Pettitt's test. The study used historical 
series of flow and precipitation corresponding to the period of 1939 to 2005, recorded at stations 02041003 and 57450000 of the hydro-meteorological network of Water National Agency (ANA), located in the municipality of Alegre-ES. Using the Pettitt test, it was possible to identify a change in the trend of October average flow since the year 1963. The average for this month increased $34.2 \%$ since 1963 . However, the rainfall series had a positive trend in August and September since the years 1967 and 1964, respectively. The increase in the October average flow may be a consequence of an increase in rainfalls in August and September; this requires further studies which may consider the influence of climate and anthropic factors in this hydrologic cycle component. Based on the results of the study, it was concluded that the Pettitt test performed satisfactorily in detecting trends in the historic series of flow and precipitation.

Keywords: average flow, Pettitt test, rain, stationarity, time series.

\section{INTRODUÇÃO}

A disponibilidade de recursos hídricos é uma questão de importância global e seu conhecimento é fundamental para o desenvolvimento da sociedade. Dentre as variáveis para avaliar a disponibilidade hídrica destaca-se a vazão de cursos d'água, a qual, nas últimas décadas tem sido influenciada expressivamente por mudanças no clima e também no uso e ocupação do solo em bacias hidrográficas. Atividades antrópicas intensivas como, por exemplo, irrigação e construção de barragens tem resultado em grandes impactos sobre a vazão, principalmente em sua magnitude (Wei et al., 2013).

$\mathrm{O}$ interesse em estudos de tendência dos componentes do ciclo hidrológico tem aumentado devido às controvérsias sobre as mudanças climáticas. De acordo com Joseph et al. (2013), a identificação de tendências sazonais de precipitação e vazão contribui para a compreensão da variabilidade climática global e é essencial para o desenvolvimento de modelos hidrológicos, previsão hidrológica e gestão dos recursos hídricos.

Hamed (2008), ao analisar a vazão anual de 35 rios em todo o mundo, verificou tendência positiva em 11 e negativa em seis considerando o nível de 5\% de significância. $\mathrm{O}$ mesmo autor, ao analisar a tendência das vazões de 12 rios dos Estados Unidos, verificou redução de vazões em um rio e aumento em três rios.

Além de verificar se a não estacionariedade de uma série hidrológica está relacionada com variações do clima ou alterações antrópicas, a realização da análise de tendência é importante na maioria das aplicações de hidrologia estatística, visto que as séries hidrológicas devem ter como pré-requisito os atributos de estacionariedade e homogeneidade (Naghettini e Pinto, 2007).

A identificação de tendência em séries históricas pode ser feita por meio de análise estatística, sendo o teste de Pettitt (Pettitt, 1979) um dos mais utilizados. Este teste não paramétrico permite confirmar a estacionariedade da série histórica, ou seja, excetuando as flutuações aleatórias, as observações são invariantes com relação à cronologia de suas ocorrências.

Com isso, o objetivo do trabalho foi analisar a tendência de séries histórica de vazão e precipitação por meio de teste estatístico não paramétrico, de forma que as informações obtidas possam ser incorporadas em estudos relacionados com a modelagem hidrológica e gestão de recursos hídricos.

\section{MATERIAL E MÉTODOS}

Foram utilizadas séries históricas de vazões e precipitações correspondentes ao período de 1939 a 2005, registradas nas estações 57450000 e 02041003, pertencente à rede 
hidrometeorológica da Agência Nacional de Águas (ANA), ambas localizadas nas coordenadas geográficas $20^{\circ} 44^{\prime} 49^{\prime}$ S e $41^{\circ} 27^{\prime} 58^{\prime} \mathrm{W}$ no município de Alegre-ES (Figura 1).

A bacia hidrográfica possui área e perímetro iguais a $2.218,0 \mathrm{~km}^{2}$ e $407,0 \mathrm{~km}$, respectivamente.

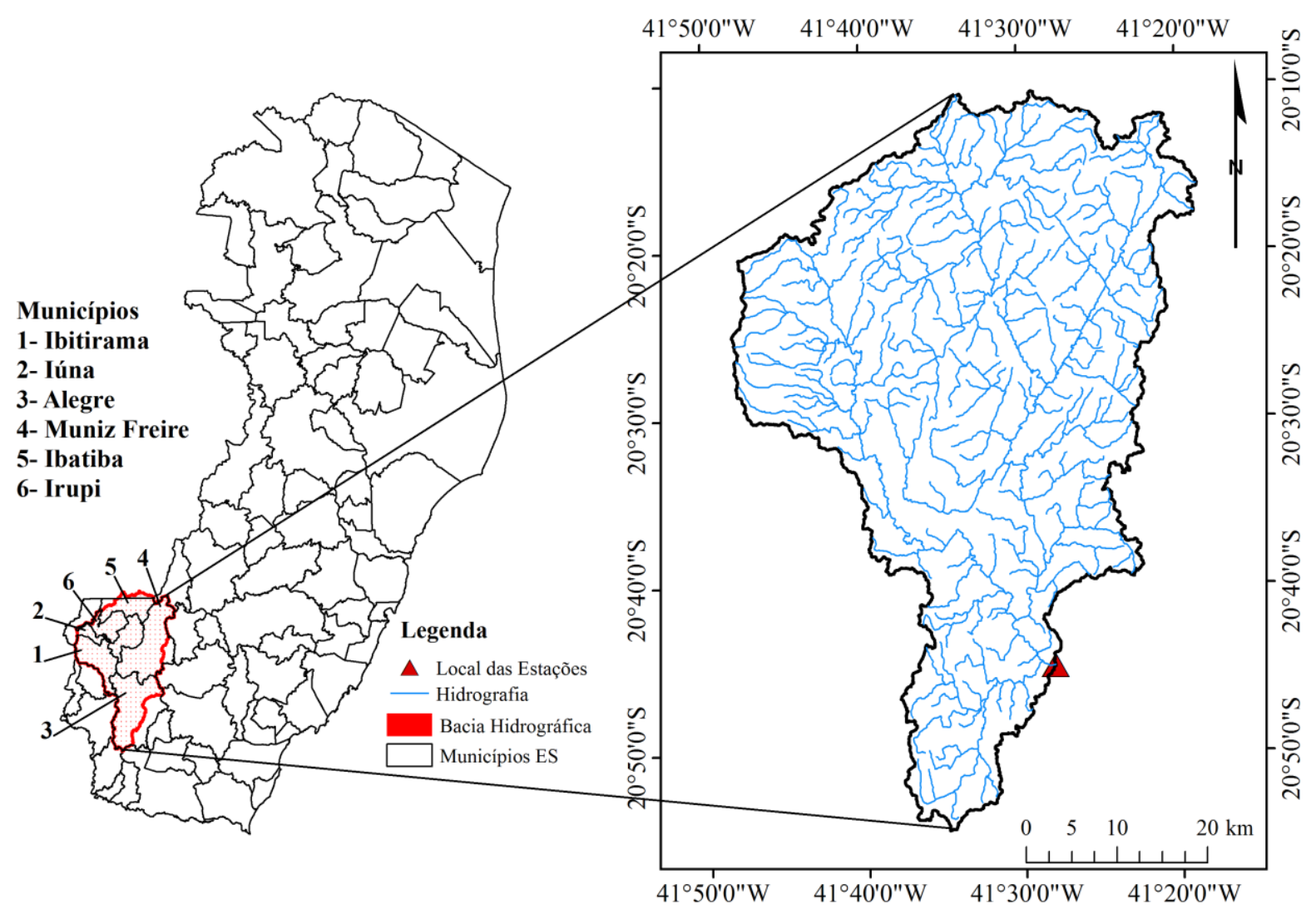

Figura 1. Localização da bacia hidrográfica e da estação fluviométrica utilizada no estudo.

Para identificação de tendência nas séries históricas de vazão média e precipitação mensal e anual foi utilizado o teste de Pettitt (Pettitt, 1979; Liu et al., 2013) ao nível de 5\% de significância. Este é um teste não paramétrico que utiliza uma versão da estatística de Mann-Whitney $U_{t, N}$, a qual verifica se duas amostras $x_{1}, \ldots, x_{t}$ e $x_{t+1}, \ldots, x_{N}$, são da mesma população ou não. A estatística de teste $U_{t, N}$ foi calculada com as Equações 1 e 2 .

$$
\mathrm{U}_{\mathrm{t}, \mathrm{N}}=\mathrm{U}_{\mathrm{t}-1, \mathrm{~N}}+\sum_{\mathrm{j}=1}^{\mathrm{N}} \operatorname{sgn}\left(\mathrm{x}_{\mathrm{t}}-\mathrm{x}_{\mathrm{j}}\right) \quad \text { para } \mathrm{t}=2,3, \ldots, \mathrm{N}
$$

em que:

$$
\operatorname{sgn}=(x)\left\{\begin{array}{c}
1 \text { se } x>0 \\
0 \text { se } x=0 \\
-1 \text { se } x<0
\end{array}\right\}
$$

A estatística de teste conta o número de vezes que o valor da primeira amostra excede o valor da segunda amostra. A hipótese nula do teste de Pettitt admite ausência de um ponto de mudança (change point) na série. A estatística $\mathrm{k}(\mathrm{t})$ representa $\mathrm{o}$ ponto de mudança t significativo no qual o valor de $\left|\mathrm{U}_{\mathrm{t}, \mathrm{N}}\right|$ é máximo e está associado a um nível de significância (P) calculado pelas Equações 3 e 4. 


$$
\begin{aligned}
& \mathrm{k}_{(\mathrm{t})}=\operatorname{máx}_{1 \leq \mathrm{t} \leq \mathrm{N}}\left|\mathrm{U}_{\mathrm{t}, \mathrm{N}}\right| \\
& \mathrm{P}=2 \mathrm{e}^{\left\{\frac{-6\left(\mathrm{~K}_{\mathrm{N}}\right)^{2}}{\left(\mathrm{~N}^{3}+\mathrm{N}^{2}\right)}\right\}}
\end{aligned}
$$

em que:

P é o nível de significância,

$\mathrm{K}_{\mathrm{N}}$ é o valor crítico e

$\mathrm{N}$ é o número de anos da série histórica.

\section{RESULTADOS E DISCUSSÃO}

Para vazões médias mensais $\left(\mathrm{m}^{3} \mathrm{~s}^{-1}\right)$, verifica-se na Figura 2 que apenas no mês de outubro foi detectada mudança de tendência na série. Neste mês, o ponto de mudança (change point) ocorreu no ano de 1963. A vazão média anual $\left(\mathrm{m}^{3} \mathrm{dia}^{-1}\right)$ apresentou estacionariedade.
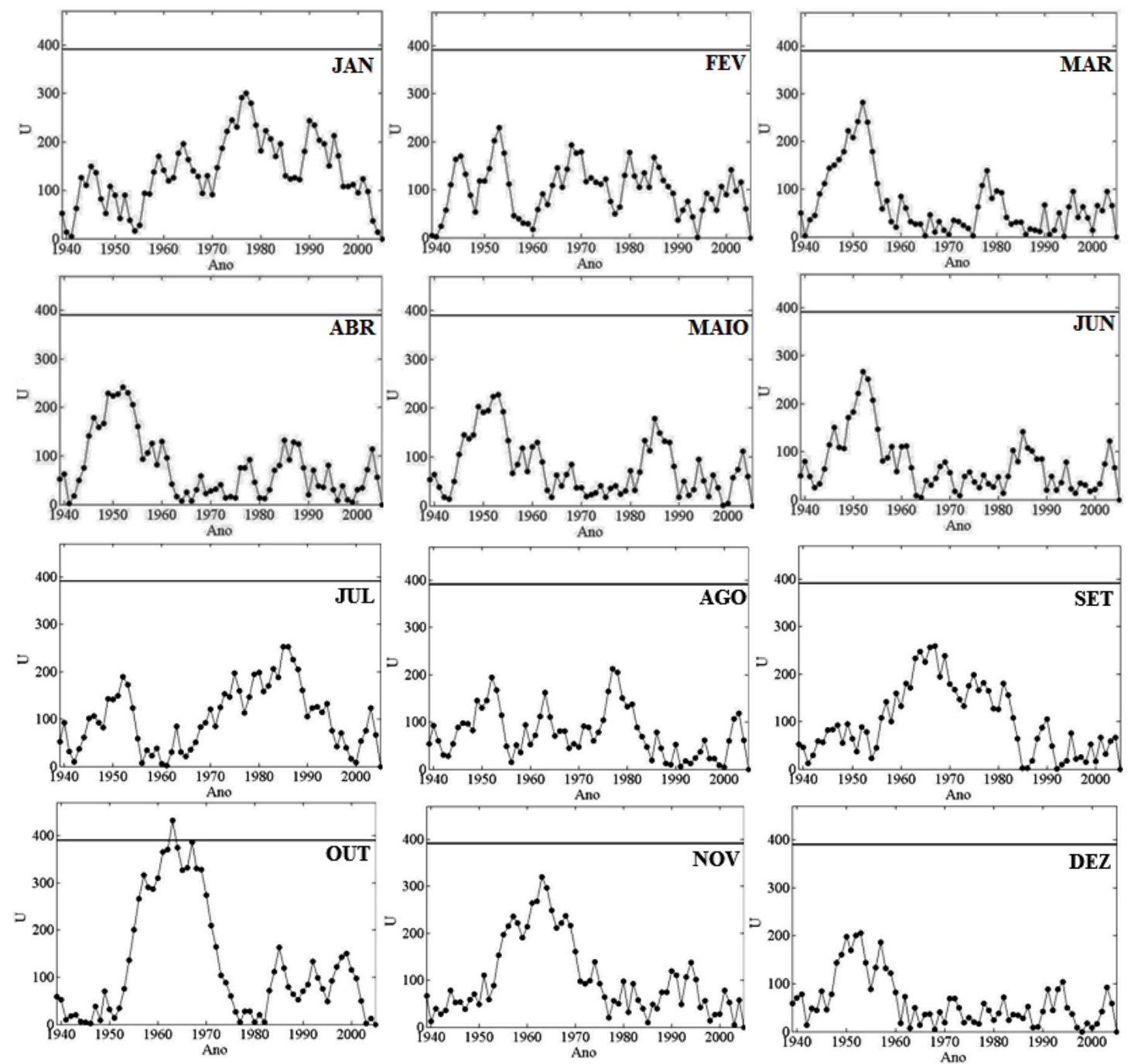

Figura 2. Resultado do teste de Pettitt para os dados de vazão média mensal $\left(\mathrm{m}^{3} \mathrm{~s}^{-1}\right)$, para o posto 57450000. 
Na Figura 3 apresenta-se a divisão da série de vazões no ano de 1963, onde se verifica que a vazão média do mês de outubro no período de 1963 a 2005 aumentou 34,2 \% em relação ao período de 1939 a 1962.

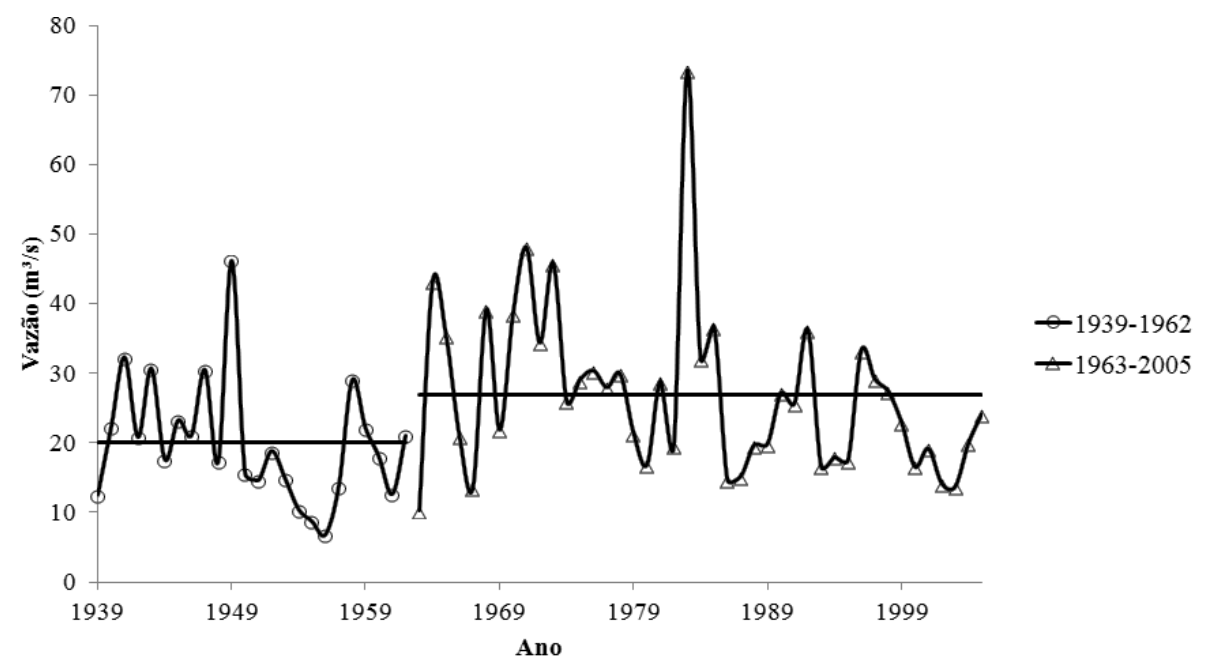

Figura 3. Série histórica da vazão média do mês de outubro, para o posto 57450000 .

Quanto as prováveis precipitações mensal e anual no estado do Espírito Santo, foram identificados por Uliana et al. (2013) dois períodos distintos: o primeiro entre outubro e abril que concentra grande parte da precipitação e o segundo entre maio e setembro com decréscimo acentuado da pluviosidade. Por meio da análise da distribuição espacial da precipitação obtida pelos autores, verifica-se que o final do período seco e o início do período chuvoso ocorrem nos meses de setembro e outubro respectivamente. O solo neste período encontra-se com baixa umidade e com máxima capacidade de infiltração e retenção de água.

$\mathrm{Na}$ Figura 4 são apresentados os resultados para a precipitação mensal (mm mês ${ }^{-1}$ ) no estado do Espírito Santo. Verifica-se na Figura 4 que nos meses de agosto e setembro foram detectadas mudanças de tendência na série, cujos pontos de mudança foram os anos de 1967 e 1964, respectivamente. Já a precipitação anual apresentou estacionariedade.

O aumento da vazão média do mês de outubro pode estar associado também a mudanças no uso e ocupação do solo que tenham reduzido a capacidade de infiltração da água no solo, causando o consequente aumento do escoamento superficial. Tucci e Clarke (1997) realizaram revisão sobre os impactos da cobertura vegetal no escoamento e verificaram, em grande parte dos estudos, que a vazão média aumenta com a redução da cobertura de florestas na bacia hidrográfica.

Com o teste de Pettitt é possível afirmar apenas que a vazão ou precipitação associada a determinado período apresenta tendência significativa, sendo necessário realizar estudos adicionais para identificação das causas deste comportamento. Estes estudos são complexos, visto ser grande o número de combinações entre os fatores envolvidos.

Outro ponto importante a ser considerado é a influência dos moduladores climáticos na precipitação e nas vazões dos cursos d'água. Wei et al. (2013) analisou o impacto da monção de verão do leste asiático (EASM) e do El Niño - Oscilação Sul (ENOS) na mudança das vazões do rio Chang Jiang e concluiu que: eventos relativamente fracos de EASM e de La Niña podem induzir aumento na vazão; e eventos relativamente fortes de EASM e El Niño podem causar redução da vazão. Com isso, o estudo de frequência destes fatores climáticos torna-se também item relevante para justificativa da mudança de tendência nas séries históricas de vazão. 

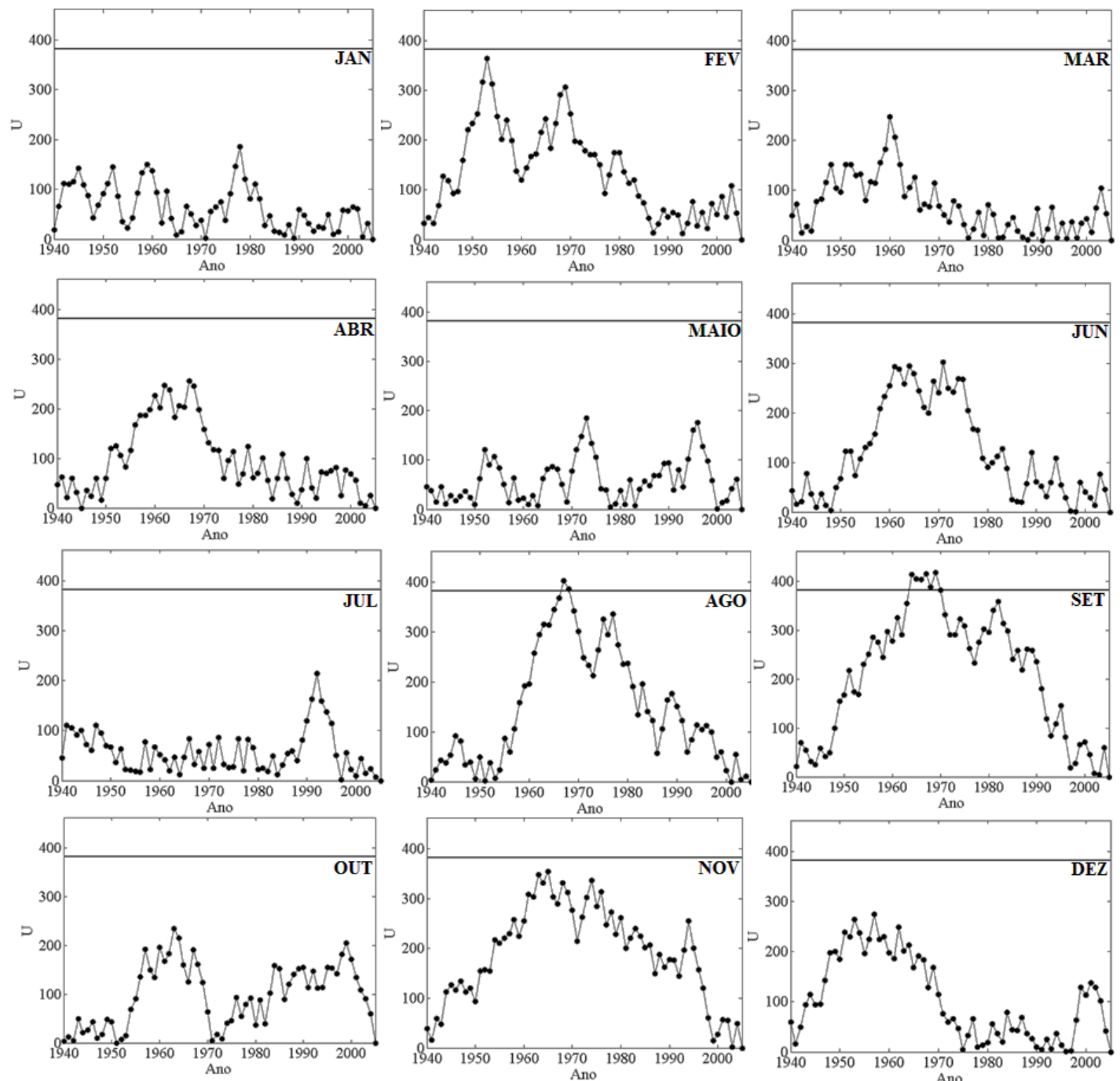

Figura 4. Resultado do teste de Pettitt para os dados de precipitação mensal (mm mês ${ }^{-1}$ ), para a estação 02041003.

Por meio da divisão da série de precipitação no ano de 1967 para o mês de agosto e no ano de 1964 para o mês de setembro foi possível verificar que a precipitação média aumentou $50,8 \%$ e $61,2 \%$ respectivamente (Figura 5). O aumento da vazão média do mês de outubro pode ser consequência do aumento da precipitação nestes dois meses antecedentes.
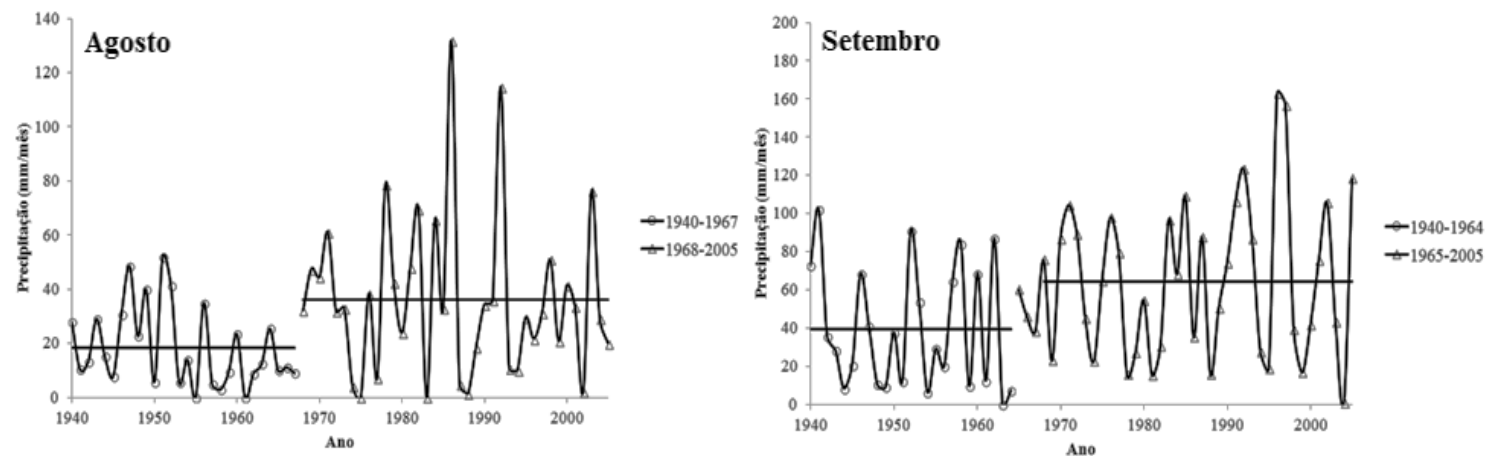

Figura 5. Séries históricas de precipitação dos meses de agosto e setembro, para a estação 02041003. 


\section{CONCLUSÕES}

O teste de Pettitt possibilitou a identificação de tendência significativa nas séries históricas de vazões e precipitações mensais, bem como do ponto de mudança. Na série de vazões médias foi identificada tendência positiva no mês de outubro com ponto de mudança no ano de 1963. Nas séries de precipitação ocorreu tendência positiva nos meses de agosto e setembro e os pontos de mudança foram os anos de 1967 e 1964, respectivamente.

$\mathrm{O}$ aumento da precipitação nos meses de agosto e setembro, dentre outros possíveis fatores, pode ter causado influência no incremento da vazão média do mês de outubro.

\section{AGRADECIMENTOS}

Os autores agradecem a Fundação de Amparo à Pesquisa do Estado de Minas Gerais (FAPEMIG) e ao Conselho Nacional de Desenvolvimento Científico e Tecnológico (CNPq) pela concessão das bolsas de doutorado e iniciação científica, bem como a Agência Nacional de Águas (ANA) pela disponibilidade dos dados hidrológicos.

\section{REFERÊNCIAS}

HAMED, K. H. Trend detection in hydrologic data: The Mann-Kendall trend test under the scaling hypothesis. Journal of Hydrology, v. 349, n. 3-4, p. 350-363, 2008. http://dx.doi.org/10.1016/j.jhydrol.2007.11.009

JOSEPH, J. F.; FALCON, H. E.; SHARIF, H. O. Hydrologic Trends and Correlations in South Texas River Basins: 1950-2009. Journal of Hydrologic Engineering, v. 18, n. 2, p. 1653-1662, 2013. http://dx.doi.org/10.1061/(ASCE)HE.1943-5584.0000709

LIU, X.; DAI, X.; ZHONG, Y.; LI, J.; WANG, P. Analysis of changes in the relationship between precipitation and streamflow in the Yiluo River, China. Theoretical and Applied Climatology, Vienna, v. 114, n. 1-2, p. 183-191, 2013. http://dx.doi.org/10.1007/s00704-013-0833-0

NAGUETTINI, M.; PINTO, E. J. A. Hidrologia estatística. Belo Horizonte: CPRM, 2007. $552 \mathrm{p}$.

PETTITT, A. N. A Non-Parametric Approach to the Change-Point Problem. Applied Statistics, v. 28, n. 2, p. 126-135, 1979. http://www.jstor.org/stable/2346729

TUCCI, E. M.; CLARKE, R. T. Impactos das mudanças da cobertura vegetal no escoamento: revisão. Revista Brasileira de Recursos Hídricos, v. 2, n. 1, p. 135-152, 1997.

ULIANA, E. M.; REIS, E. F.; SILVA, J. G. F.; XAVIER, A. C. Precipitação Mensal e Anual Provável para o Estado do Espírito Santo. Irriga, Botucatu, v. 18, n. 1, p. 139-147, 2013. http://irriga.fca.unesp.br/index.php/irriga/article/view/493/344

WEI, W.; CHANG, Y.; DAI, Z. Streamflow changes of the Changjiang (Yangtze) River in the recent 60 years: Impacts of the East Asian summer monsoon, ENSO, and human activities. Quaternary International, p. 1-10, 2013.

http://dx.doi.org/10.1016/j.quaint.2013.10.064 